Karol Dziedziul and Barbara Wolnik (Gdańsk)

\title{
NOTE ON UNIVERSAL ALGORITHMS FOR LEARNING THEORY
}

Abstract. We study the universal estimator for the regression problem in learning theory considered by Binev et al. This new approach allows us to improve their results.

1. Introduction. S. Cucker and S. Smale [1] determined the scope of learning theory. We present a general approach which corresponds to [2] and [3]. The problem is the following. Let $X=[0,1]^{d}$ and $Y=[-A, A]$. On the product space $Z=X \times Y$ there is an unknown probability Borel measure $\varrho$. We shall assume that the marginal probability measure $\varrho_{X}(S)=\varrho(S \times Y)$ on $X$ is a Borel measure. We have

$$
d \varrho(x, y)=d \varrho(y \mid x) d \varrho_{X}(x) .
$$

We are given the data $\mathbf{z} \subset Z$ of $m$ independent random observations $z_{j}=\left(x_{j}, y_{j}\right), j=1, \ldots, m$, identically distributed according to $\varrho$. We are interested in estimating the regression function

$$
f_{\varrho}(x):=\int_{Y} y d \varrho(y \mid x)
$$

in $L^{2}\left(X, \varrho_{X}\right)$ norm which will be denoted by $\|\cdot\|$.

To do it let $\mathbf{M}=\left\{M_{v}\right\}_{v \in T}$ denote any family of measurable functions on $X$ such that for all $v \in T$,

$$
0 \leq M_{v}(x) \leq 1, \quad x \in X,
$$

and

$$
\sum_{v \in T} M_{v}(x)=1, \quad x \in X
$$

2000 Mathematics Subject Classification: 68T05, 41A36, 41A45, 62G05.

Key words and phrases: nonparametric regression, learning theory. 
An example is the family $\left\{\chi_{I}\right\}_{I \in T}$, where $\chi_{I}$ denotes the indicator function of $I$ and $\{I: I \in T\}$ is any partition of $X$ (in [2] the sets $I$ are dyadic cubes). Another example is obtained if we consider a triangulation $T$ of $X$ with vertices $\{v\}_{v \in T}$ and the corresponding system of functions $\left\{M_{v}\right\}_{v \in T}$ which are continuous on $X$, linear on each component of this triangulation and

$$
M_{v}(w)= \begin{cases}1 & \text { for vertices } w=v, \\ 0 & \text { for } w \neq v .\end{cases}
$$

It is not hard to check that the family $\left\{M_{v}\right\}_{v \in T}$ satisfies (1) and (2).

Now for a given family $\mathbf{M}$ we define the operator

$$
Q_{\mathbf{M}} f(x)=\sum_{v \in T} c_{v}(f) M_{v}(x),
$$

where

$$
c_{v}(f)=\frac{\alpha_{v}(f)}{\varrho_{v}}, \quad \alpha_{v}(f)=\int_{X} f M_{v} d \varrho_{X}, \quad \varrho_{v}=\int_{X} M_{v} d \varrho_{X},
$$

and the estimator

$$
f_{\mathbf{z}}(x)=\sum_{v \in T} c_{v}(\mathbf{z}) M_{v}(x)
$$

where

$$
c_{v}(\mathbf{z})=\frac{\alpha_{v}(\mathbf{z})}{\varrho_{v}(\mathbf{z})}, \quad \alpha_{v}(\mathbf{z})=\frac{1}{m} \sum_{j=1}^{m} y_{j} M_{v}\left(x_{j}\right), \quad \varrho_{v}(\mathbf{z})=\frac{1}{m} \sum_{j=1}^{m} M_{v}\left(x_{j}\right) .
$$

If $\varrho_{v}=0$ then we define $c_{v}=0$, and if $\varrho_{v}(\mathbf{z})=0$ then we put $c_{v}(\mathbf{z})=0$. Note also that $E \alpha_{v}(\mathbf{z})=\alpha_{v}$ (here and subsequently, $\alpha_{v}:=\alpha_{v}\left(f_{\varrho}\right), c_{v}:=c_{v}\left(f_{\varrho}\right)$ ) and $E \varrho_{v}(\mathbf{z})=\varrho_{v}$. Moreover

$$
\operatorname{Var}\left(y M_{v}(x)\right) \leq \int_{Z} y^{2} M_{v}^{2}(x) d \varrho(x, y) \leq A^{2} \int_{X} M_{v}^{2}(x) d \varrho_{X}(x),
$$

hence

$$
\begin{aligned}
& \operatorname{Var}\left(y M_{v}(x)\right) \leq A^{2} \int_{X} M_{v}(x) d \varrho_{X}(x)=A^{2} \varrho_{v}, \\
& \operatorname{Var}\left(M_{v}(x)\right) \leq E\left(M_{v}(x)\right)^{2} \leq E\left(M_{v}(x)\right)=\varrho_{v} .
\end{aligned}
$$

Therefore by Bernstein's inequality we have, for any $\varepsilon>0$,

$$
\begin{aligned}
& \operatorname{Prob}\left\{\left|\alpha_{v}-\alpha_{v}(\mathbf{z})\right| \geq \varepsilon\right\} \leq 2 \exp \left(-\frac{3 m \varepsilon^{2}}{6 A^{2} \varrho_{v}+4 A \varepsilon}\right), \\
& \operatorname{Prob}\left\{\left|\varrho_{v}-\varrho_{v}(\mathbf{z})\right| \geq \varepsilon\right\} \leq 2 \exp \left(-\frac{3 m \varepsilon^{2}}{6 \varrho_{v}+2 \varepsilon}\right) .
\end{aligned}
$$

The main result of this paper is 
Theorem 1.1. For any family $\mathbf{M}$,

$$
E\left\|Q_{\mathbf{M}} f_{\varrho}-f_{\mathbf{z}}\right\|^{2}=O\left(\frac{N}{m}\right)
$$

where $N=|T|$.

The new idea of the proof presented below allows us to improve the result from [2] (in Corollary 2.2 of [2] the above expectation is bounded by $O((N / m) \log N))$.

Proof. By (1), (2) and the convexity of the square functions we have

$$
\begin{aligned}
E\left\|Q_{\mathbf{M}} f_{\varrho}-f_{\mathbf{z}}\right\|^{2} & \leq \int_{X} \sum_{v \in T} E\left|c_{v}-c_{v}(\mathbf{z})\right|^{2} M_{v}(x) d \varrho_{X}(x) \\
& =\sum_{v \in T} E\left|c_{v}-c_{v}(\mathbf{z})\right|^{2} \varrho_{v} .
\end{aligned}
$$

Note that if $\varrho_{v}=0$ then $E \varrho_{v}(\mathbf{z})=0$, hence $\varrho_{v}(\mathbf{z})=0 \varrho^{m}$-a.e. Consequently,

$$
E\left\|Q_{\mathbf{M}} f_{\varrho}-f_{\mathbf{z}}\right\|^{2} \leq \sum_{v \in T, \varrho_{v}>0} E\left|c_{v}-c_{v}(\mathbf{z})\right|^{2} \varrho_{v}
$$

Fix $v$ such that $\varrho_{v}>0$. We can write

$$
E\left|c_{v}-c_{v}(\mathbf{z})\right|^{2}=\int_{\varrho_{v}(\mathbf{z})>0}\left|c_{v}-c_{v}(\mathbf{z})\right|^{2}+\int_{\varrho_{v}(\mathbf{z})=0}\left|c_{v}\right|^{2} .
$$

Note that if $\varrho_{v}(\mathbf{z})=0 \varrho^{m}$-a.e. then $M_{v}\left(x_{j}\right)=0$ for all $j$, hence $\alpha_{v}(\mathbf{z})=0$ $\varrho^{m}$-a.e. Thus

$$
E\left|c_{v}-c_{v}(\mathbf{z})\right|^{2}=\int_{\varrho_{v}(\mathbf{z})>0}\left|c_{v}-c_{v}(\mathbf{z})\right|^{2}+\int_{\varrho_{v}(\mathbf{z})=0}\left|\frac{\alpha_{v}-\alpha_{v}(\mathbf{z})}{\varrho_{v}}\right|^{2} .
$$

For $b \neq 0$ and $t \neq 0$ we use the simple inequality

$$
\left|\frac{a}{b}-\frac{s}{t}\right| \leq \frac{1}{|b|}|a-s|+\frac{|s|}{|b t|}|t-b|
$$

to get

$$
\left|\frac{a}{b}-\frac{s}{t}\right|^{2} \leq 2 \frac{|a-s|^{2}}{b^{2}}+2 \frac{1}{b^{2}} \frac{s^{2}}{t^{2}}|t-b|^{2},
$$

which in particular gives

$$
\left|\frac{a_{v}}{\varrho_{v}}-\frac{a_{v}(\mathbf{z})}{\varrho_{v}(\mathbf{z})}\right|^{2} \leq 2 \frac{\left|a_{v}-a_{v}(\mathbf{z})\right|^{2}}{\varrho_{v}^{2}}+2\left(\frac{a_{v}(\mathbf{z})}{\varrho_{v}(\mathbf{z})}\right)^{2} \frac{\left|\varrho_{v}-\varrho_{v}(\mathbf{z})\right|^{2}}{\varrho_{v}^{2}} .
$$

For $\varrho_{v}(\mathbf{z})>0$ we have

$$
\frac{\alpha_{v}(\mathbf{z})^{2}}{\varrho_{v}(\mathbf{z})^{2}} \leq A^{2}
$$

thus

$$
E\left|c_{v}-c_{v}(\mathbf{z})\right|^{2} \leq \frac{3}{m \varrho_{v}^{2}} \operatorname{Var}\left(y M_{v}(x)\right)+\frac{2 A^{2}}{m \varrho_{v}^{2}} \operatorname{Var}\left(M_{v}(x)\right) .
$$


Consequently,

$$
E\left\|Q_{T} f_{\varrho}-f_{\mathbf{z}}\right\|^{2} \leq C \sum_{v \in T} \frac{1}{m \varrho_{v}^{2}}\left(\operatorname{Var}\left(y M_{v}(x)\right)+\operatorname{Var}\left(M_{v}(x)\right)\right) \varrho_{v} .
$$

By (3) and (4) we get

$$
E\left\|Q_{T} f_{\varrho}-f_{\mathbf{z}}\right\|^{2} \leq O\left(\sum_{v \in T} \frac{1}{m}\right)=O\left(\frac{N}{m}\right)
$$

and this finishes the proof.

Note that if we take $N=m^{1 /(1+2 s)}$ for fixed $s>0$ then

$$
E\left\|Q_{\mathbf{M}} f_{\varrho}-f_{\mathbf{z}}\right\|^{2}=O\left(\frac{1}{m}\right)^{2 s /(1+2 s)} .
$$

To unify the linear and nonlinear approach in estimation let us introduce the sets $\mathcal{A}^{s}$ similar to the definition given in [2]. We have $f \in \mathcal{A}^{s}, s>0$ (in fact it makes sense to consider $0<s \leq 2)$ if $f \in L^{2}\left(\varrho_{X}\right)$ and there is $C$ such that for all $N$ there is a family $\mathbf{M}=\left\{M_{v}\right\}_{v \in T}$ with properties (1) and (2) such that $N=|T|$ and

$$
\left\|f-Q_{\mathbf{M}} f\right\| \leq C N^{-s} .
$$

By Theorem 1.2, (9) and (10), and since

$$
E\left\|f_{\varrho}-f_{\mathbf{z}}\right\|^{2} \leq 2 E\left\|f_{\varrho}-Q_{\mathbf{M}} f_{\varrho}\right\|^{2}+2 E\left\|Q_{\mathbf{M}} f_{\varrho}-f_{\mathbf{z}}\right\|^{2},
$$

we get the optimal rate of estimation (see [4]). This approach improves the rate of estimation in [2].

Theorem 1.2. Let $f_{\varrho} \in \mathcal{A}^{s}$ and let $\mathbf{M}$ be the family from the definition of the space $\mathcal{A}^{s}$ such that $N=|T|=\left[m^{1 /(1+2 s)}\right]$. Then

$$
E\left\|f_{\varrho}-f_{\mathbf{z}}\right\|^{2}=O\left(\frac{1}{m}\right)^{2 s /(1+2 s)} .
$$

Finally, we will give a general version of Theorem 2.1 in [2]. Our proof is analogous but partially simplified, so we present it for the sake of completeness. We improve the constant in estimation.

Theorem 1.3. For any family $\mathbf{M}$ and any $\eta>0$,

$$
\operatorname{Prob}\left\{\left\|Q_{\mathbf{M}} f_{\varrho}-f_{\mathbf{z}}\right\|>\eta\right\} \leq 4 N e^{-c m \eta^{2} / N},
$$

where $N:=|T|$ and $c$ depends only on $A$.

Proof. By the convexity of the square function we have

$$
\begin{aligned}
\left\|Q_{\mathbf{M}} f_{\varrho}-f_{\mathbf{z}}\right\|^{2} & \leq \int_{X} \sum_{v \in T}\left|c_{v}-c_{v}(\mathbf{z})\right|^{2} M_{v}(x) d \varrho_{X}(x) \\
& =\sum_{v \in T}\left|c_{v}-c_{v}(\mathbf{z})\right|^{2} \varrho_{v} .
\end{aligned}
$$


This gives

$$
\begin{aligned}
\operatorname{Prob}\left\{\left\|Q_{\mathbf{M}} f_{\varrho}-f_{\mathbf{z}}\right\|>\eta\right\} & \leq \operatorname{Prob}\left\{\sum_{v \in T}\left|c_{v}-c_{v}(\mathbf{z})\right|^{2} \varrho_{v}>\eta^{2}\right\} \\
& \leq \sum_{v \in T} \operatorname{Prob}\left\{\left|c_{v}-c_{v}(\mathbf{z})\right|>\frac{\eta}{\sqrt{N \varrho_{v}}}\right\} .
\end{aligned}
$$

Note that

$$
\operatorname{Prob}\left\{\left|c_{v}-c_{v}(\mathbf{z})\right|>\frac{\eta}{\sqrt{N \varrho_{v}}}\right\}=0
$$

provided $\varrho_{v} \leq \eta^{2} / 4 A^{2} N$. To see this it is enough to transform this assumption to the form $\eta / \sqrt{N \varrho_{v}} \geq 2 A$ and recall that $\left|c_{v}\right|$ and $\left|c_{v}(\mathbf{z})\right|$ are less than $A$.

Therefore we can write

$$
\operatorname{Prob}\left\{\left\|Q_{\mathbf{M}} f_{\varrho}-f_{\mathbf{z}}\right\|>\eta\right\} \leq \sum_{v: \varrho_{v}>\eta^{2} / 4 A^{2} N} \operatorname{Prob}\left\{\left|c_{v}-c_{v}(\mathbf{z})\right|>\frac{\eta}{\sqrt{N \varrho_{v}}}\right\} .
$$

To estimate the last sum, note that if

$$
\left|\alpha_{v}(\mathbf{z})-\alpha_{v}\right| \leq \frac{\varrho_{v} \eta}{4 \sqrt{N \varrho_{v}}}
$$

and

$$
\left|\varrho_{v}(\mathbf{z})-\varrho_{v}\right| \leq \frac{\varrho_{v} \eta}{4 A \sqrt{N \varrho_{v}}}
$$

then (we know that $\varrho_{v}>\eta^{2} / 4 A^{2} N$ )

$$
\left|\varrho_{v}(\mathbf{z})-\varrho_{v}\right| \leq \frac{\varrho_{v} \eta}{4 A \sqrt{N \frac{\eta^{2}}{4 A^{2} N}}}=\frac{1}{2} \varrho_{v}
$$

(this gives in particular $\left|\varrho_{v}(\mathbf{z})\right| \geq \frac{1}{2} \varrho_{v}$ ), and using (7) we get

$$
\begin{aligned}
\left|c_{v}(\mathbf{z})-c_{v}\right| & =\left|\frac{\alpha_{v}(\mathbf{z})}{\varrho_{v}(\mathbf{z})}-\frac{\alpha_{v}}{\varrho_{v}}\right| \\
& \leq \frac{1}{\varrho_{v}(\mathbf{z}) \mid}\left|\alpha_{v}(\mathbf{z})-\alpha_{v}\right|+\frac{\left|\alpha_{v}\right|}{\left|\varrho_{v}(\mathbf{z})\right| \varrho_{v}}\left|\varrho_{v}(\mathbf{z})-\varrho_{v}\right| \\
& \leq \frac{1}{\frac{1}{2} \varrho_{v}} \cdot \frac{\varrho_{v} \eta}{4 \sqrt{N \varrho_{v}}}+\frac{A}{\frac{1}{2} \varrho_{v}} \cdot \frac{\varrho_{v} \eta}{4 A \sqrt{N \varrho_{v}}}=\frac{\eta}{\sqrt{N \varrho_{v}}} .
\end{aligned}
$$

Therefore

$$
\begin{aligned}
& \operatorname{Prob}\left\{\left|c_{v}-c_{v}(\mathbf{z})\right|>\frac{\eta}{\sqrt{N \varrho_{v}}}\right\} \\
& \quad \leq \operatorname{Prob}\left\{\left|\alpha_{v}(\mathbf{z})-\alpha_{v}\right|>\frac{\varrho_{v} \eta}{4 \sqrt{N \varrho_{v}}}\right\}+\operatorname{Prob}\left\{\left|\varrho_{v}(\mathbf{z})-\varrho_{v}\right|>\frac{\varrho_{v} \eta}{4 A \sqrt{N \varrho_{v}}}\right\} .
\end{aligned}
$$

If we first use (5), (6) and then the fact that $\eta / \sqrt{N \varrho_{v}} \leq 2 A$, we finally get 


$$
\begin{aligned}
\operatorname{Prob}\left\{\| Q_{\mathbf{M}} f_{\varrho}-\right. & \left.f_{\mathbf{z}} \|>\eta\right\} \\
\leq & \sum_{v: \varrho_{v}>\eta^{2} / 4 A^{2} N}\left(2 \exp \left(-\frac{3 m \eta^{2}}{16 N\left(6 A^{2}+A \frac{\eta}{\sqrt{N \varrho_{v}}}\right)}\right)\right. \\
& \left.+2 \exp \left(-\frac{3 m \eta^{2}}{16 A^{2} N\left(6+\frac{1}{2 A} \cdot \frac{\eta}{\sqrt{N \varrho_{v}}}\right)}\right)\right) \\
\leq & \sum_{v: \varrho_{v}>\eta^{2} / 4 A^{2} N} 2\left(\exp \left(-\frac{3}{128} \cdot \frac{m \eta^{2}}{N A^{2}}\right)+\exp \left(-\frac{3}{112} \cdot \frac{m \eta^{2}}{N A^{2}}\right)\right) \\
\leq & 4 N \exp \left(-\frac{3}{128 A^{2}} \cdot \frac{m \eta^{2}}{N}\right),
\end{aligned}
$$

which completes the proof of (11) with $c=3 / 128 A^{2}$.

\section{References}

[1] S. Cucker and S. Smale, On the mathematical foundations of learning, Bull. Amer. Math. Soc. 39 (2001), 1-49.

[2] P. Binev, A. Cohen, W. Dahmen, R. DeVore and V. Temlyakov, Universal algorithms for learning theory. Part I: piecewise constant functions, J. Machine Learning Res. 6 (2005), 1297-1321.

[3] - , - - - - - - Universal algorithms for learning theory. Part II: piecewise constant functions, preprint.

[4] R. DeVore, G. Kerkyacharian, D. Picard and V. Temlyakov, Approximation methods for supervised learning, Found. Comput. Math. 1 (2006), 3-58.

Karol Dziedziul

Faculty of Applied Mathematics

Gdańsk University of Technology

Narutowicza 11/12

80-952 Gdańsk, Poland

E-mail: kdz@mifgate.pg.gda.pl
Barbara Wolnik Institute of Mathematics

Gdańsk University

Wita Stwosza 57

80-952 Gdańsk, Poland E-mail: Barbara.Wolnik@math.univ.gda.pl

Received on 16.10.2006;

revised version on 15.2.2007 